\title{
Minocycline-Induced Polyarteritis Nodosa Presenting With Testicular Pain: A Case Report and Selected Review of the Literature
}

\author{
Bridget Lang Findlay, ${ }^{\mathrm{a},}$, Young Suk Kwon ${ }^{\mathrm{a}}$ e, f, Joshua Sterling ${ }^{\mathrm{a}}$, Aisha Fatima ${ }^{\mathrm{b}}$, \\ Lakshmi N. Moorthyc ${ }^{c}$, George Halko ${ }^{d}$, Evita Sadimin ${ }^{\mathrm{b}}$, Joseph Barone ${ }^{\mathrm{a}}$
}

\begin{abstract}
Polyarteritis nodosa (PAN) is a systemic necrotizing vasculitis that affects small and medium-sized arteries, leading to aneurysm formation and ischemia in different organs of the body. Although not common, testicular manifestations have been documented. We discuss a case of 18-year-old male with minocycline-induced systemic PAN who presented with unilateral testicular pain. The definitive diagnosis was made after testicular biopsy demonstrating focally necrotizing vasculitis affecting small and medium vessels. Following the diagnosis he was discharged on prednisone, and was started on rituximab by his rheumatologist. $\mathrm{He}$ is clinically much improved during his surveillance follow-up visits with his rheumatologist and urologist. Minocycline-induced polyarteritis nodosa can present with constitutional symptoms and testicular pain among patients using the acne treatment. Prompt diagnosis is essential to ensure proper treatment and prevention of complications. Optimal management of these patients requires close collaboration amongst urologists and rheumatologists for accurate tissue diagnosis and close surveillance.
\end{abstract}

Keywords: Minocycline; Polyarteritis nodosa; Testis; Vasculitis

\section{Introduction}

Testicular pain is a non-specific finding which may be mechan-

Manuscript submitted March 7, 2018, accepted March 14, 2018

a'Division of Urology, Department of Surgery, Rutgers Robert Wood Johnson Medical School, New Brunswick, NJ, USA

bDepartment of Pathology and Laboratory Medicine, Rutgers Robert Wood Johnson Medical School, New Brunswick, NJ, USA

'Department of Pediatric Rheumatology, Rutgers Robert Wood Johnson Medical School, New Brunswick, NJ, USA

${ }^{\mathrm{d} D e p a r t m e n t}$ of Rheumatology, AtlantiCare Regional Medical Center, Atlantic City, NJ, USA

eEqual contribution

${ }^{\mathrm{f} C}$ orresponding Author: Young Suk Kwon, Division of Urology, Department of Surgery, Rutgers Robert Wood Johnson Medical School, 1 Robert Wood Johnson Place, MEB Suite 584, New Brunswick, NJ 08902, USA.

Email: yk411@rutgers.edu

doi: https://doi.org/10.14740/wjnu337w ical, infectious, oncologic, or rheumatologic in its etiologies. Polyarteritis nodosa (PAN) is a critical rheumatologic condition that can present with genitourinary manifestations. This condition has a slight male predominance, and its prevalence is estimated to be 31 cases/million [1]. Its peak incidence occurs in the fourth to sixth decade of life. Our patient, 18 years old, is certainly younger than many of the reported cases. Urologic symptoms may appear as the earliest manifestation of the systemic disease. Previous reports have shown that patients diagnosed with PAN have testicular pain with increased vascularity seen on scrotal US. Moreover, hematuria is also seen in $15 \%$ of patients, and orchitis/epididymitis is seen in $2-18 \%$ of patients with PAN [2]. Here we present an 18-year-old male with acute on chronic testicular pain. The case presentation is followed by a selected review of the literature on this topic.

\section{Case Report}

An 18-year-old male with 9-month history of intermittent chronic left testicular pain and swelling associated with weight loss, arthralgias, and myalgias, presented with an acute onset of left testicular pain of 1-week duration. On initial presentation to his rheumatologist, he had been taking minocycline $100 \mathrm{mg}$ twice daily for 2 years for acne. The medication was discontinued by his rheumatologist and changed to azithromycin for management of his acne. He tested positive for ANA (1:160) and anti-histone antibody ( 2.7 units) at that time, and was started on prednisone $60 \mathrm{mg}$ daily by his rheumatologist, which was tapered down over the course of 6 months to $5 \mathrm{mg}$ every other day. Initial specific ANCA testing was negative for anti-MPO and anti-PR3. One week prior to admission, he presented to the emergency department with testicular pain and swelling, and was discharged with an increase in his prednisone dose to 20 $\mathrm{mg}$ once daily. One day prior to admission, the testicular pain intensified with a new onset of left lower quadrant abdominal pain. Associated symptoms included dysuria, hesitancy, and gross hematuria.

On exam, the left testicle was mildly tender to palpation without any masses. The left epididymis was firm and focally tender, and there was a palpable left varicocele. The right testicle was non-tender without any masses. His labs were remarkable for hemoglobin of $10.1 \mathrm{~g} / \mathrm{L}$, hematuria on urinalysis with 44 red blood cells per high power field (RBCs/HPF), elevated 


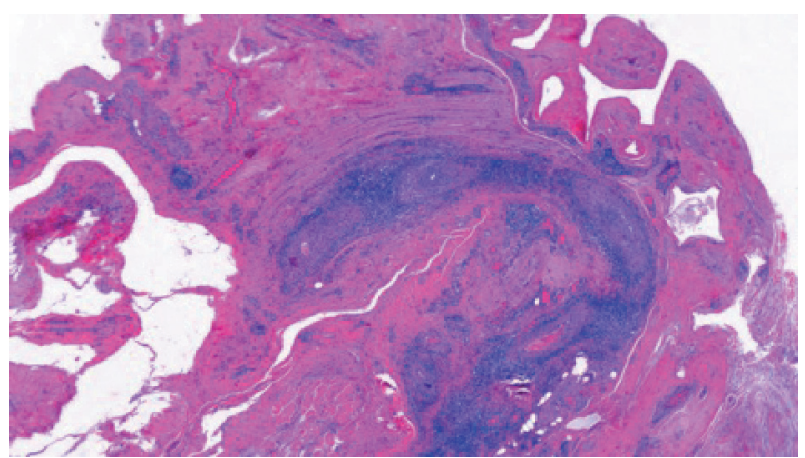

Figure 1. Left paratesticular tissue with perivascular infiltrates containing of lymphocytes and plasma cells (H\&E, $20 \times$ original magnification).

erythrocyte sedimentation rate (ESR) and C-reactive protein (CRP), positive ANA (> 1:1280) and positive ANCA (MPO positive 2.3). Scrotal ultrasound demonstrated heterogeneous appearing testes with increased vascularity in bilateral testes and epididymis, and a possible left varicocele.

On admission he was continued on the same dose of oral prednisone until biopsy results were obtained for definitive diagnosis. His abdominal pain and hematuria had completely resolved, and testicular pain was improving each day. Given the testicular symptoms, the patient underwent bilateral testicular and paratesticular tissue biopsy to rule out systemic vasculitis. The bilateral paratesticular biopsies demonstrated focally necrotizing vasculitis affecting small and medium vessels with fibrinoid necrosis (Fig. 1,2). Staining for IgG and IgG4 revealed rare scattered IgG4 positive plasma cells among a population of IgG positive cells. Immunofluorescence was otherwise negative for an immune complex mediated vasculitis as the complement and fibrinogen staining related to focal, active areas of inflammation involved by fibrinoid necrosis. Testicular tissue was negative for inflammation, infarction, or parenchymal vasculitis.

Additional workup for suspected vasculitis included a MRA of the chest and abdomen, with attention to the renal arteries. Our patient showed aortic root dilation on MRA of the chest with the absence of renal aneurysm or infarct on MRA of the abdomen. Although this was not a new finding, it is important to continue to monitor with his cardiologist. After biopsy results were back, the patient's dose of prednisone was increased to $60 \mathrm{mg}$ daily to reduce inflammation and he resumed follow-up with rheumatology as an outpatient. He was advised to avoid minocycline for treatment of acne in the future. On his follow-up visit with his rheumatologist 1 month after discharge, he was given a $2 \mathrm{~g}$ infusion of rituximab and prednisone was tapered down to $15 \mathrm{mg}$ daily. The plan is to continue rituximab infusions every 4 - 6 months for a total of three to four cycles in addition to the prednisone. Two months following discharge, he is asymptomatic and has not had any recurrence of his testicular pain, abdominal pain, or hematuria.

\section{Discussion}

The differential diagnosis of acute testicular pain commonly

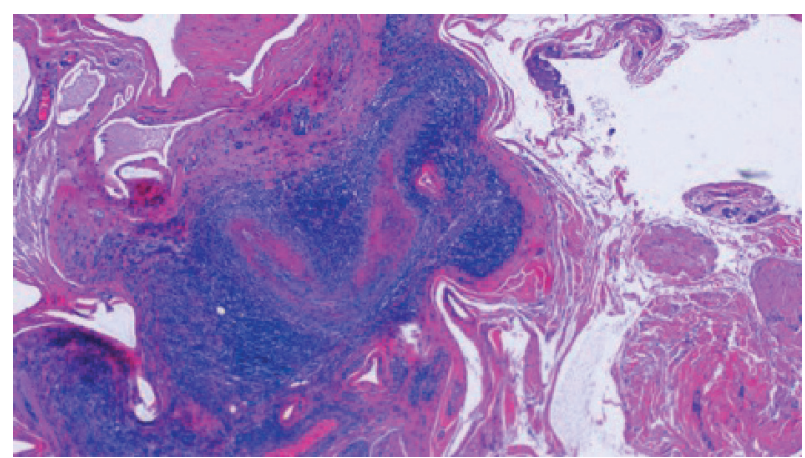

Figure 2. Left paratesticular tissue with focal necrotizing vasculitis of small and medium sized vessels and perivascular infiltrates containing of lymphocytes and plasma cells (H\&E, $40 \times$ original magnification).

includes testicular torsion, appendicular torsion, orchitis, epididymitis, or tumor. Many clinicians often neglect to consider testicular pain as a presenting symptom of vasculitis. To our best knowledge, our patient is the youngest of all the patients who had testicular pain as an initial manifestation of systemic PAN (Table 1, [3-18]). In addition to testicular pain, our patient displayed diffuse myalgias, unexplained weight loss meeting the diagnosing criteria for PAN [19].

Typically, pathogenesis of PAN is idiopathic, although hepatits $\mathrm{B}$, hepatitis $\mathrm{C}$, and HIV are associated with the vasculitis [2]. In addition, there are documented cases of minocycline-induced PAN [3]. It is hypothesized that myeloperoxidase enzyme oxidizes minocycline into reactive metabolites, which can trigger the induction of pANCA and lead to an autoimmune response [3]. In consistency with this finding, our patient had a history of minocycline use for acne treatment, and also demonstrated MPO-ANCA positivity on laboratory testing. It should be noted that patients with systemic PAN are typically negative for ANCA, and its positivity in the setting of necrotizing vasculitis should strongly suggest the presence of another form of vasculitis, including microscopic polyangiitis [2].

While our patient's symptoms were highly suggestive of vasculitic etiology, histopathologic finding is the most definitive diagnosis of PAN. Active lesions of the affected arteries in the PAN typically demonstrate areas of fibrinoid necrosis and neutrophilic infiltration, as seen in the biopsy slide of our patient [2]. This differs from granulomatosis with polyangiitis, formerly known as Wegener granulomatosis, which demonstrates necrotizing granulomatous inflammation of small and medium-size vessels in addition to PR3-ANCA positivity. Another consideration in the differential of testicular pain among systemic vasculitis is Henoch-Schonlein purpura (HSP). However, HSP is associated with the presence of immune complexes containing $\operatorname{IgA}$ along with its cutaneous manifestation of palpable purpura. Given the high index of suspicion for vasculitis in the absence of palpable testicular mass, orchiectomy was avoided as testicular biopsy was sufficient to establish the diagnosis.

Management options for testicular pain in the setting of PAN are not clearly established. While there are reports of testicular PAN treated with orchiectomy alone without any recurrence of disease after 1 to 3 years of follow-up $[11,13,15,16$, 


\begin{tabular}{|c|c|c|c|c|c|c|c|}
\hline 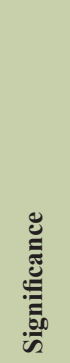 & 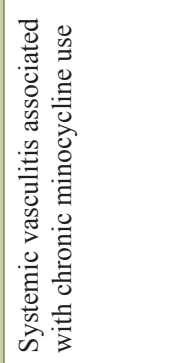 & 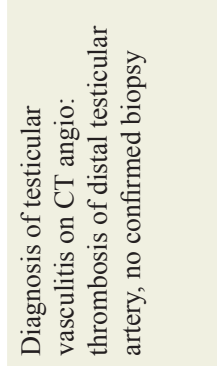 & 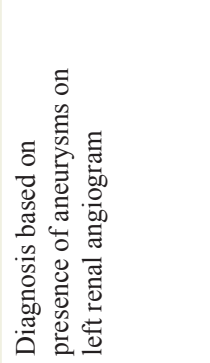 & 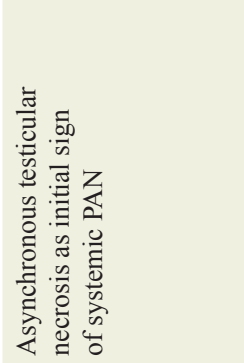 & 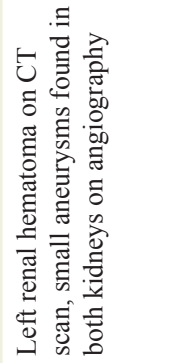 & 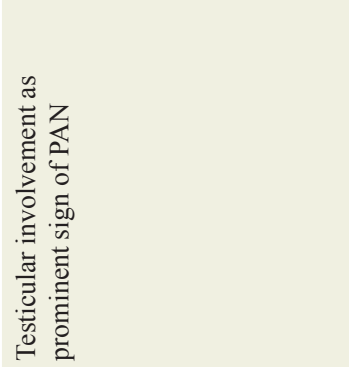 & 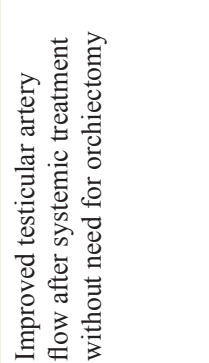 \\
\hline 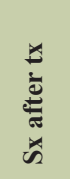 & 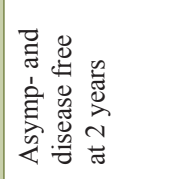 & 言 & 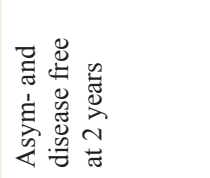 & 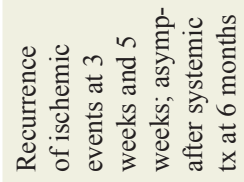 & & 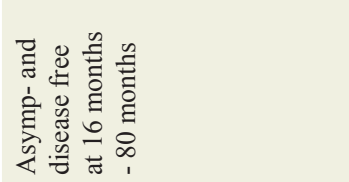 & $\overleftrightarrow{z}$ \\
\hline 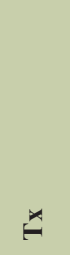 & 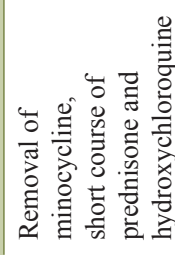 & 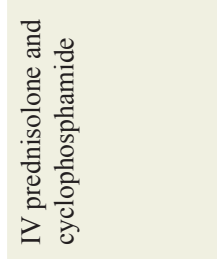 & 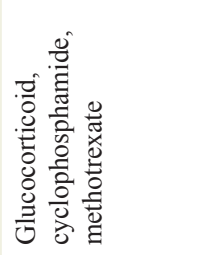 & 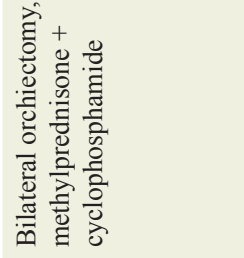 & 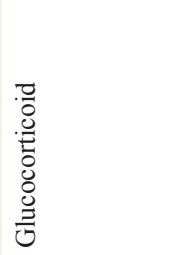 & 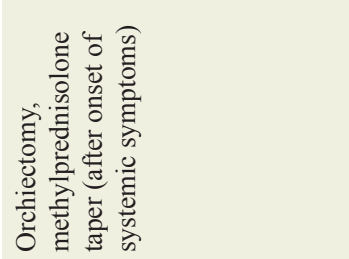 & 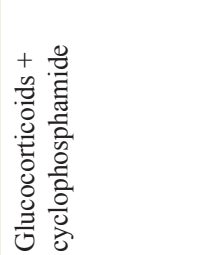 \\
\hline 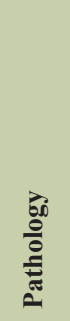 & 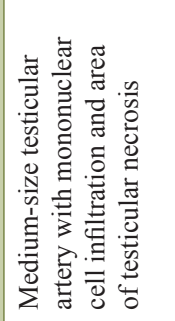 & 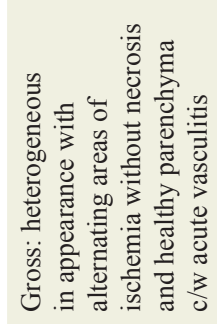 & & 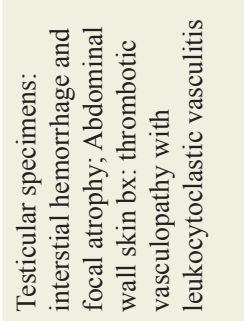 & & 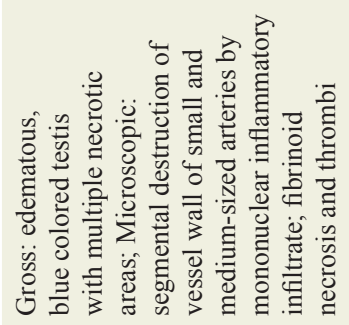 & \\
\hline 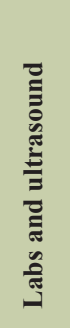 & 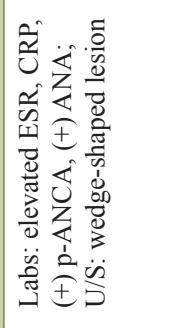 & 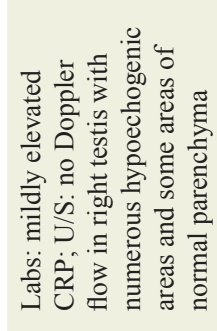 & 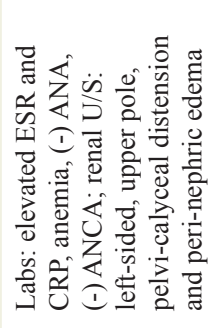 & 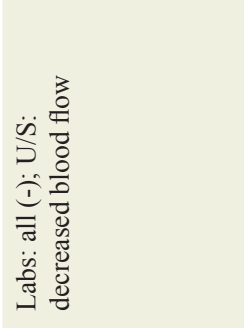 & 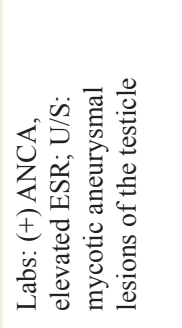 & 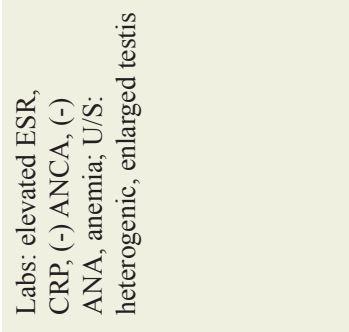 & 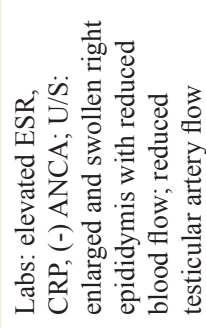 \\
\hline U & 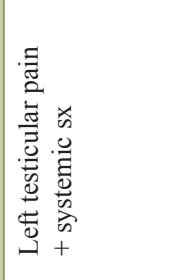 & 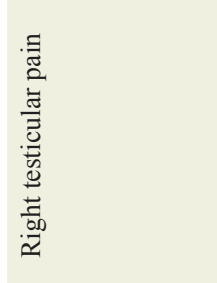 & 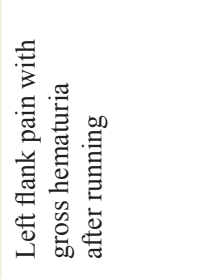 & 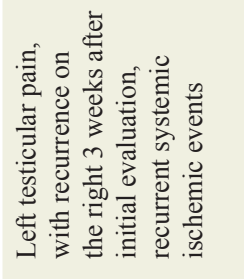 & 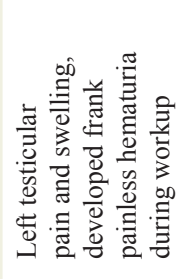 & 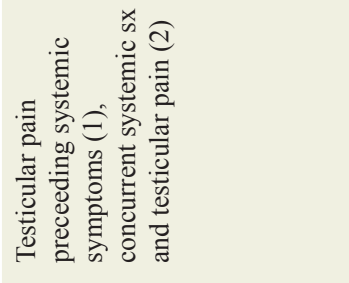 & 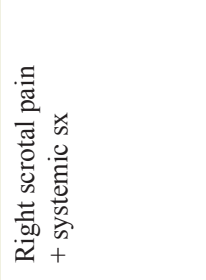 \\
\hline 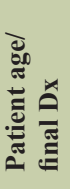 & 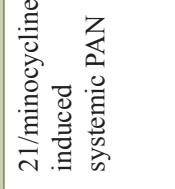 & 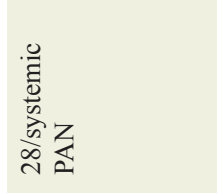 & 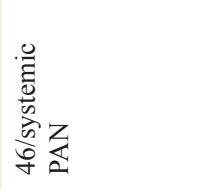 & 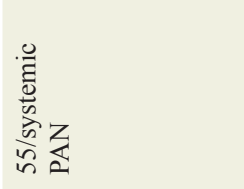 & 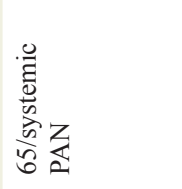 & 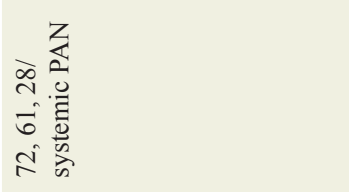 & 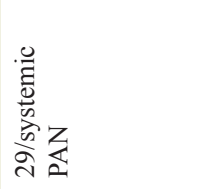 \\
\hline 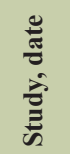 & 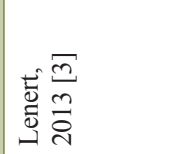 & 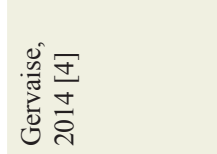 & 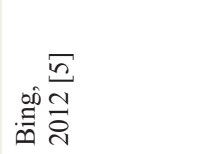 & 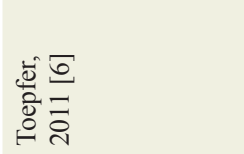 & 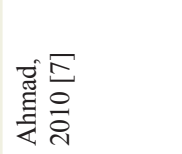 & 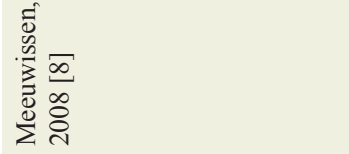 & 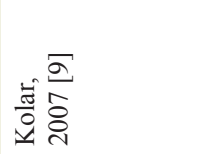 \\
\hline
\end{tabular}




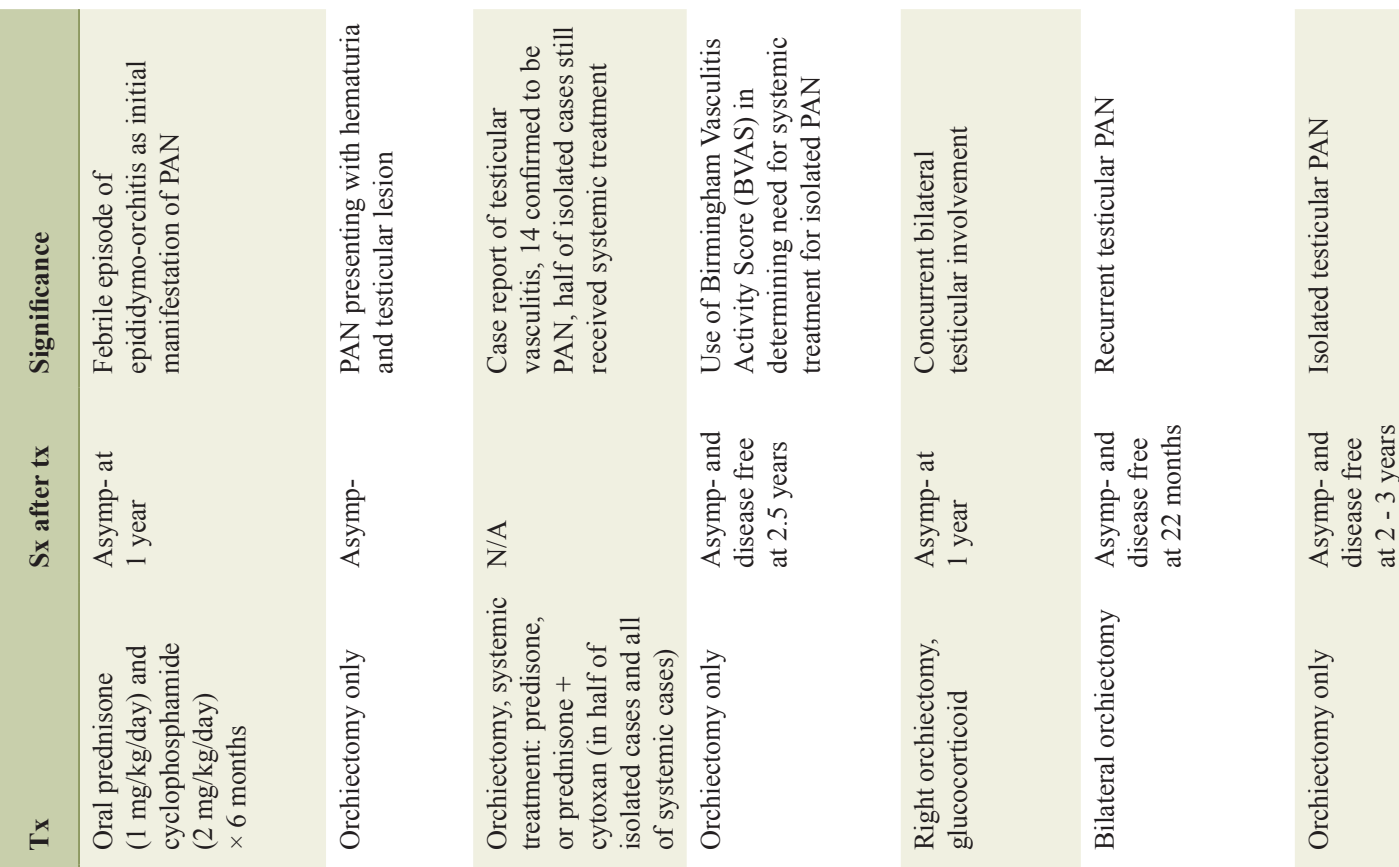

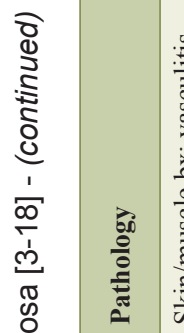
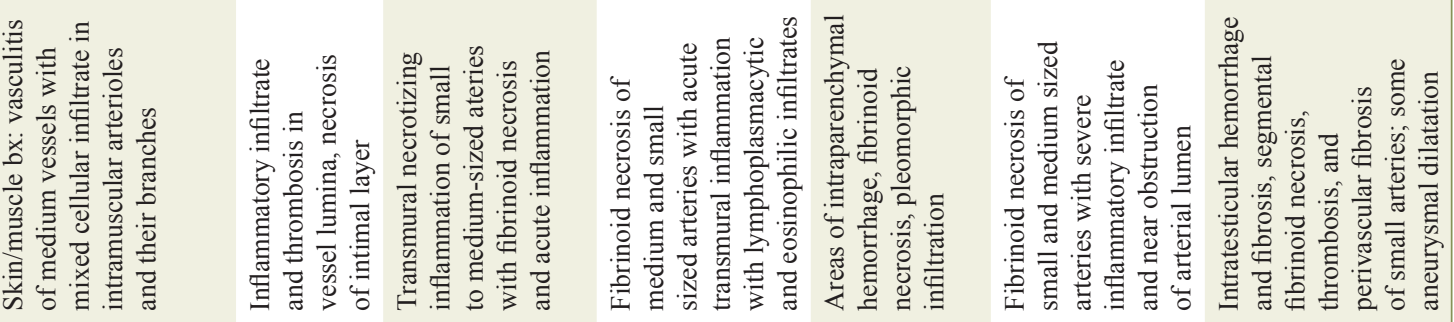

政

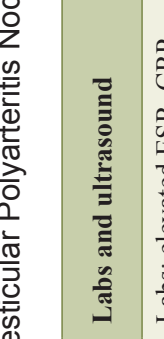

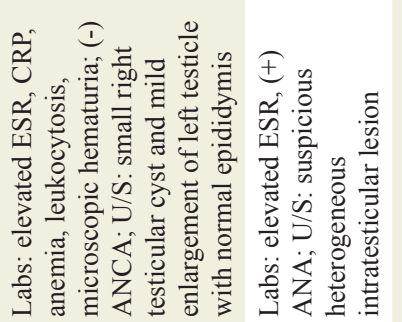

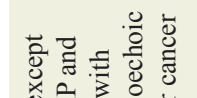

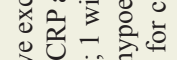
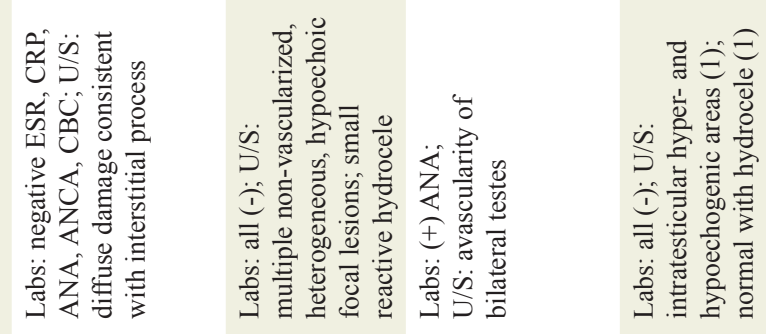

\%

ญ

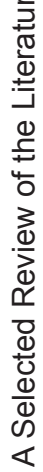
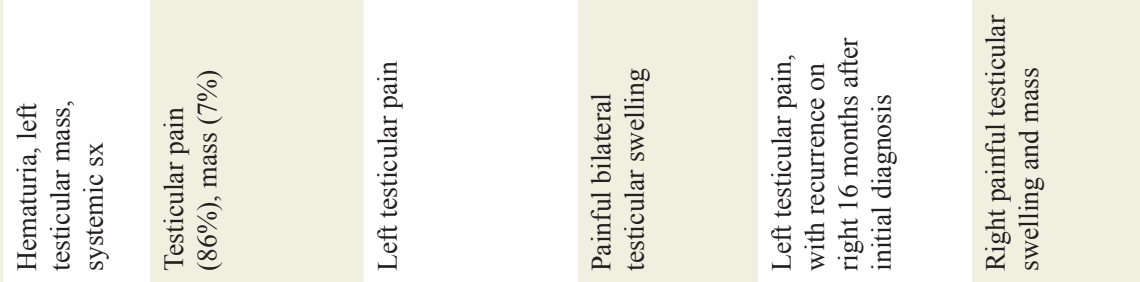

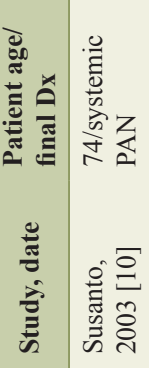
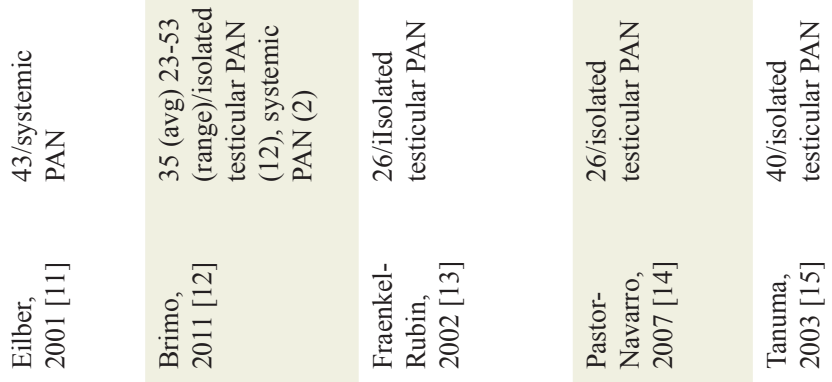

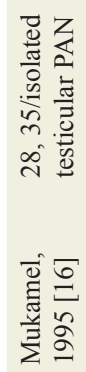




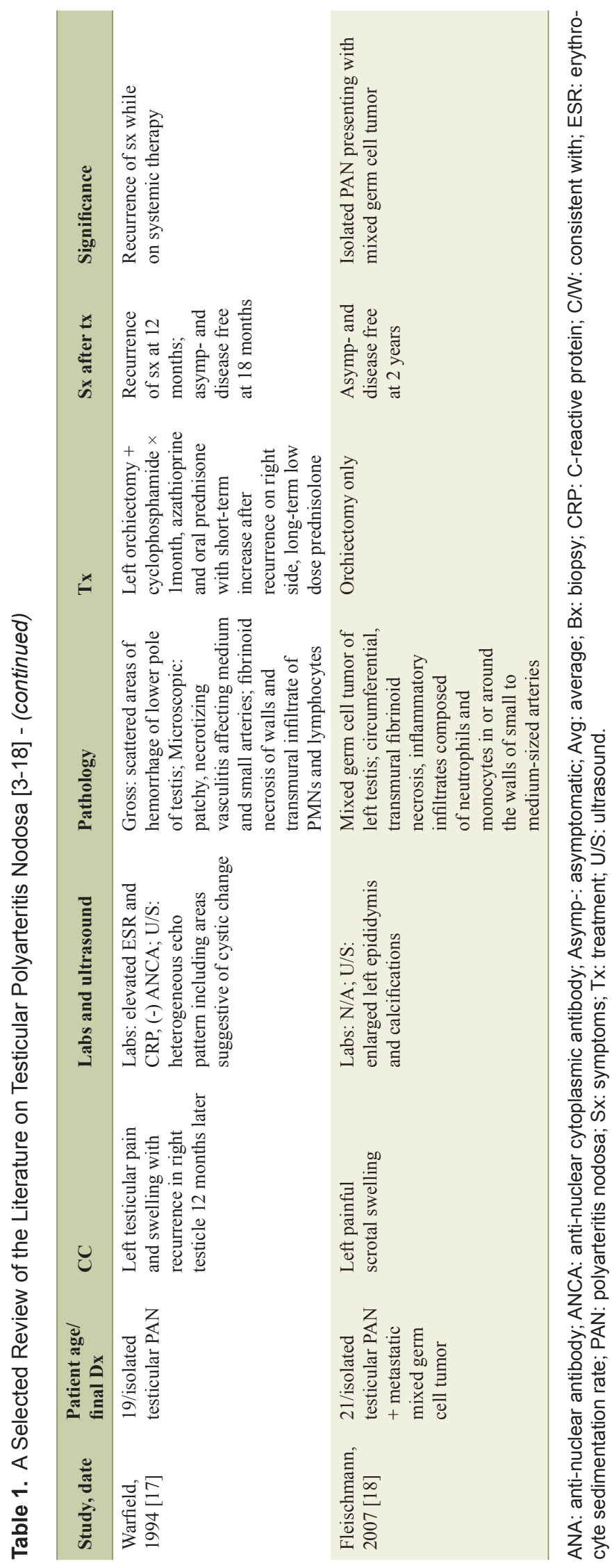

20], there is also a case of minocycline-induced systemic PAN with a short course of prednisone and hydroxychloroquine alone for complete symptom resolution [3]. To better characterize and quantify the severity of symptoms, Fraenkel-Rubin et al used the Birmingham Vasculitis Activity Scale (BVAS), a 56 point assessment of disease activity, in order to determine if a patient with testicular vasculitis needs systemic therapy [13, 21]. Based on this scale, a low BVAS score indicates a localized disease while high BVAS means a systemic disease which requires steroid therapy with or without orchiectomy. Our patient displayed systemic symptoms and scored moderately high on BVAS, and his testicular pain had been well managed with prednisone $60 \mathrm{mg}$ once a day taper without any surgical intervention at his 1-month follow-up.

Surveillance is paramount to monitor for symptom recurrence and progression. Although our patient's hematuria had completely resolved at the time of testicular biopsy, further systemic signs and recurrence of testicular pain would mandate both additional imaging work and possible orchiectomy, respectively. Lastly, due to the complexity of this clinical condition, close communication and collaborative efforts between urology and rheumatology are crucial in monitoring symptoms and coordinating treatment options.

\section{Conclusions}

Minocycline-induced PAN can present with constitutional symptoms and testicular pain among patients using the acne treatment. Although it is uncommon, it is important to consider systemic vasculitis as a possible diagnosis when a patient presents with acute testicular pain along with musculoskeletal symptoms. Optimal management of these patients requires close collaboration amongst urologists and rheumatologists for accurate tissue diagnosis and close surveillance.

\section{Conflict of Interest}

There is no conflict of interest to disclose.

\section{Grant Support}

This research did not receive any specific grant from funding agencies in the public, commercial, or not-for-profit sectors.

\section{Abbreviations}

ANA: anti-nuclear antibody; ANCA: anti-nuclear cytoplasmic antibody; Avg: average; BP: blood pressure; BUN: blood urea nitrogen; BVAS: Birmingham Vasculitis Activity Scale; Bx: biopsy; CBC: complete blood count; CRP: C-reactive protein; CT: computed tomography; C/W: consistent with; ESR: erythrocyte sedimentation rate; HSP: Henoch-Schonlein purpura; MPO: myeloperoxidase; MRA: magnetic resonance angiography; MRI: magnetic resonance imaging; PAN: polyarteritis 
nodosa; RBCs/HPF: Red blood cells per high power field; Sx: symptoms; Tx: treatment; U/S: ultrasound

\section{References}

1. Forbess L, Bannykh S. Polyarteritis nodosa. Rheumatic diseases clinics of North America. 2015;41(1):33-46, vii.

2. Hernandez-Rodriguez J, Alba MA, Prieto-Gonzalez S, Cid MC. Diagnosis and classification of polyarteritis nodosa. J Autoimmun. 2014;48-49:84-89.

3. Lenert P, Icardi M, Dahmoush L. ANA (+) ANCA (+) systemic vasculitis associated with the use of minocycline: case-based review. Clin Rheumatol. 2013;32(7):10991106.

4. Gervaise A, Junca-Laplace C, Naulet P, Pernin M, Portron Y, Lapierre-Combes M. Unilateral testicular vasculitis in polyarteritis nodosa mimicking a testicular torsion. Diagn Interv Imaging. 2014;95(6):615-616.

5. Bing A, Rich C, Keanie JY, Ralston SH, Stewart GD. Polyarteritis nodosa presenting as haematuria following strenuous exercise. BMJ Case Rep. 2012;2012.

6. Toepfer NJ, Lountzis NI, Ugoeke JC, Ferringer TC. Polyarteritis nodosa with bilateral asynchronous testicular necrosis: a case report. Case Rep Urol. 2011;2011:465353.

7. Ahmad MS, Saha A, Reaich D, Naisby GP, West AF. Polyarteritis nodosa presenting with frank hematuria. Can Urol Assoc J. 2010;4(6):E172-174.

8. Meeuwissen J, Maertens J, Verbeken E, Blockmans D. Case reports: testicular pain as a manifestation of polyarteritis nodosa. Clin Rheumatol. 2008;27(11):14631466.

9. Kolar P, Schneider U, Filimonow S, Burmester GR, Buttgereit F. Polyarteritis nodosa and testicular pain: ultrasonography reveals vasculitis of the testicular artery. Rheumatology (Oxford). 2007;46(8):1377-1378.

10. Susanto CR, Fedder G, Looijen-Salamon MG. Acute, painful, and swollen testicle as the presenting feature in polyarteritis nodosa. Eur J Intern Med. 2003;14(7):441443.
11. Eilber KS, Freedland SJ, Rajfer J. Polyarteritis nodosa presenting as hematuria and a testicular mass. J Urol. 2001;166(2):624.

12. Brimo F, Lachapelle J, Epstein JI. Testicular vasculitis: a series of 19 cases. Urology. 2011;77(5):1043-1048.

13. Fraenkel-Rubin M, Ergas D, Sthoeger ZM. Limited polyarteritis nodosa of the male and female reproductive systems: diagnostic and therapeutic approach. Ann Rheum Dis. 2002;61(4):362-364.

14. Pastor-Navarro H, Broseta-Viana L, Donate-Moreno MJ, Pastor-Guzman JM, Lorenzo-Romero JG, Segura-Martin M, Salinas-Sanchez AS, et al. Isolated testicular polyarteritis nodosa. Urology. 2007;70(1):178 e177-178.

15. Tanuma Y, Oda T, Yokoo A, Ito S, Takeuchi K. Recurrent polyarteritis nodosa limited to the testis. J Urol. 2003;170(5):1953.

16. Mukamel E, Abarbanel J, Savion M, Konichezky M, Yachia D, Auslaender L. Testicular mass as a presenting symptom of isolated polyarteritis nodosa. Am J Clin Pathol. 1995;103(2):215-217.

17. Warfield AT, Lee SJ, Phillips SM, Pall AA. Isolated testicular vasculitis mimicking a testicular neoplasm. J Clin Pathol. 1994;47(12):1121-1123.

18. Fleischmann A, Studer UE. Isolated polyarteritis nodosa of the male reproductive system associated with a germ cell tumor of the testis: a case report. Cardiovasc Pathol. 2007;16(6):354-356.

19. Lightfoot RW, Jr., Michel BA, Bloch DA, Hunder GG, Zvaifler NJ, McShane DJ, Arend WP, et al. The American College of Rheumatology 1990 criteria for the classification of polyarteritis nodosa. Arthritis Rheum. 1990;33(8):1088-1093.

20. Hernandez-Rodriguez J, Tan CD, Koening CL, Khasnis A, Rodriguez ER, Hoffman GS. Testicular vasculitis: findings differentiating isolated disease from systemic disease in 72 patients. Medicine (Baltimore). 2012;91(2):75-85.

21. Mukhtyar C, Lee R, Brown D, Carruthers D, Dasgupta B, Dubey S, Flossmann O, et al. Modification and validation of the Birmingham Vasculitis Activity Score (version 3). Ann Rheum Dis. 2009;68(12):1827-1832. 